Pacific

Journal of

Mathematics

SUR LES OPÉRATEURS NILPOTENTS À IMAGES DES ITÉRÉS FERMÉES DANS UN ESPACE DE BANACH

ABDELKHALEK FAOUZI

Volume $200 \quad$ No. 1

September 2001 


\title{
SUR LES OPÉRATEURS NILPOTENTS À IMAGES DES ITÉRÉS FERMÉES DANS UN ESPACE DE BANACH
}

\author{
ABDElKhaleK FAOUZi \\ This work is dedicated to the memory of \\ Professor M. El Oufir.
}

\begin{abstract}
Nous montrons que, pour un opérateur linéaire $A$ nilpotent à images des itérés fermées dans un espace de Banach $E$, tout sous-espace de $E$ de codimension finie contient un sousespace réduisant pour $A$ de codimension finie. D'autre part, par le biais de l'étude des sous-espaces réduisants minimaux contenant un sous-espace donné, nous prouvons que toute extension continue d'un opérateur nilpotent à images des itérés fermées par un opérateur nilpotent défini en dimension finie est aussi à images des itérés fermées. D'autres résultats sur les opérateurs nilpotents à images des itérés fermées sont établis.
\end{abstract}

\section{Introduction.}

Soient $E$ un espace de Banach complexe et $\mathcal{B}(E)$ l'algèbre des opérateurs linéaires continus sur $E$. Par sous-espace de $E$ nous entendons sous-espace vectoriel fermé de $E$. Pour $A \in \mathcal{B}(E)$, nous désignons par Lat $A$ le treillis des sous-espaces de $E$ invariants pour $A$. L'opérateur $A \in \mathcal{B}(E)$ est dit à images des itérés fermées si $\operatorname{Im} A^{k}=A^{k}(E)$ est fermée pour tout entier positif $k$. Un résultat assez remarquable de M. El Oufir ([4], Chapitre IV, Théorème 3) affirme qu'un opérateur nilpotent est à images des itérés fermées si et seulement si tout sous-espace de $E$ de dimension finie est contenu dans un sous-espace réduisant pour $A$ de dimension finie. Ce théorème, ainsi que l'étude en dimension finie des extensions d'opérateurs linéaires (voir [3] et [5]) ont motivé notre intérêt pour l'étude des extensions d'opérateurs nilpotents à images des itérés fermées par des opérateurs nilpotents "de dimension finie" (i.e., définis sur des espaces de dimension finie). De ce fait, il s'est avéré nécessaire pour nous d'établir certaines propriétés sur les opérateurs nilpotents à images des itérés fermées. Ainsi, comme conséquence non triviale du théorème d'El Oufir mentionné ci-dessus, nous montrons que si $A \in \mathcal{B}(E)$ est nilpotent à images des itérés fermées, alors tout sous-espace invariant pour $A$ de codimension finie contient un sous-espace réduisant pour $A$ de codimension finie. La réciproque reste à étudier. D'autre part, nous démontrons, toujours pour $A \in \mathcal{B}(E)$ nilpotent 
à images des itérés fermées, que s'il existe $E_{1}, E_{2}, F_{1}, F_{2}$, dans Lat $A$ tels que $(E, A)=\left(E_{1}, A_{1}\right) \oplus\left(F_{1}, B_{1}\right)=\left(E_{2}, A_{2}\right) \oplus\left(F_{2}, B_{2}\right)$ où $A_{i}=A \mid E_{i}$ et $B_{i}=B \mid F_{i}$ (pour $\left.i=1,2\right)$ et si $\left(E_{1}, A_{1}\right)$ et $\left(E_{2}, A_{2}\right)$ sont isomorphes et de dimension finie, alors $\left(F_{1}, B_{1}\right)$ et $\left(F_{2}, B_{2}\right)$ sont isomorphes.

Dans une autre partie, nous montrons que toute extension continue d'un opérateur nilpotent à images des itérés fermées par un opérateur nilpotent "de dimension finie" est aussi à images des itérés fermées. Un contre-exemple montre qu'en général une extension continue d'un opérateur nilpotent à images des itérés fermées n'est pas toujours à images des itérés fermées. Enfin, nous indiquons comment l'étude des extensions des opérateurs nilpotents à images des itérés fermées par des opérateurs nilpotents "de dimension finie" peut être ramenée au cas de la dimension finie. Nous traitons pour cela deux cas particuliers (assez intéressants) mais le principe reste le même à chaque fois qu'une condition d'unicité à isomorphie près est vérifiée.

\section{Notions préliminaires.}

Soient $E$ un espace de Banach sur le corps des complexes $\mathcal{C}$ et $A \in \mathcal{B}(E)$. On peut munir $E$ d'une structure de $\mathcal{C}[X]$-module en posant $P(x)=P(A) x$ pour tout $x \in E$ et pour tout $P \in \mathcal{C}[X]$. Nous notons $(E, A)$ le module ainsi défini.

Nos méthodes sont, pour la plupart, inspirées de la théorie des groupes abéliens (voir [6] et [7]) laquelle, interprétée en termes de modules sur un anneau principal, peut être appliquée aux opérateurs linéaires.

Si $M$ est un sous-ensemble de $E$, on notera $\operatorname{Vect}(M)$ le plus petit sousespace de $E$ contenant $M$ et $\operatorname{Vect}_{A}(M)$ le plus petit sous-espace invariant pour $A$ contenant $M$.

Si $A$ est nilpotent, on définit l'exposant $e(x)$ de $x \in E$ comme étant le plus petit entier positif tel que $A^{k} x=0$. On définit la hauteur de $x \in E$, notée $h(x)$, comme étant l'entier $k \in \mathcal{N}$ tel que $x \in A^{k} E$ et $x \notin A^{k+1} E$ avec la convention $h(0)=\infty$. On vérifie facilement les propriétés suivantes de la hauteur:

(i) $h(x+y) \geq \inf (h(x), h(y)) \quad$ (avec égalité si $h(x) \neq h(y)$ ).

(ii) $h(\alpha x)=h(x)$, pour tout $\alpha \in \mathcal{C}, \alpha \neq 0$.

(iii) $h(A x) \geq h(x)+1$.

Soit $F$ un sous-espace de $E$. On dit que $F$ est pur dans le $\mathcal{C}[X]$-module $(E, A)$ si:

$$
\forall P \in \mathcal{C}[X], \quad P(A) F=F \cap P(A) E .
$$

On a les propriétés suivantes:

Lemme 2.1. Si $F$ est pur dans $(E, A)$, alors $F$ est pur dans $(G, A)$ pour tout sous-espace $G$ invariant pour $A$ et contenant $F$.

Lemme 2.2. Tout sous-espace réduisant pour $A$ est pur dans (E, $A)$. 
Lemme 2.3. Si $E$ est de dimension finie et $A \in \mathcal{B}(E)$ est nilpotent, alors il y a équivalence entre sous-espace pur dans $(E, A)$ et sous-espace réduisant pour $A$.

Notation 2.4. Nous réservons le signe $\oplus$ à la somme directe topologique, alors que nous désignerons une somme directe algébrique par le signe $\dot{+}$. D'autre part, le signe $\cong$ désignera l'isomorphie de modules, d'espaces vectoriels ou d'espaces de Banach.

\section{Quelques propriétés des opérateurs nilpotents à images des itérés fermées.}

Lemme 3.1. Soient $E$ et $H$ deux espaces de Banach, $A$ un opérateur de $E$ dans $H$ et $F$ un sous-espace de codimension finie dans $E . S i \operatorname{Im} A=A(E)$ est fermée dans $H$, alors $\operatorname{Im}(A \mid F)=A(F)$ est fermée aussi.

Démonstration. Soit le morphisme $T$ défini de $F /(F \cap \operatorname{Ker} A)$ dans $E / \operatorname{Ker} A$ par $T(y+F \cap \operatorname{Ker} A)=y+\operatorname{Ker} A$, pour tout $y \in F$. $T$ est injectif d'image $\operatorname{Im} T=(F+\operatorname{Ker} A) / \operatorname{Ker} A$. Montrons que $\operatorname{Im} T$ est fermée dans $E / \operatorname{Ker} A$. Pour cela, il suffit que l'on montre que $F+\operatorname{Ker} A$ est fermé dans $E$. On considère le morphisme $S$ de $\operatorname{Ker} A /(F \cap \operatorname{Ker} A)$ dans $E / F$ défini par $S(z+$ $F \cap \operatorname{Ker} A)=z+F$, pour tout $z \in \operatorname{Ker} A$. $S$ est injectif, ce qui entraîne $\operatorname{dim}(\operatorname{Ker} A /(F \cap \operatorname{Ker} A)) \leq \operatorname{dim}(E / F)=\operatorname{codim} F<+\infty$. Ainsi, $F \cap \operatorname{Ker} A$ est de codimension finie dans $\operatorname{Ker} A$, donc admet un supplémentaire algébrique $G$ dans $\operatorname{Ker} A$. Par suite, $F+\operatorname{Ker} A=F+G$ avec $G$ de dimension finie, donc $F+\operatorname{Ker} A$ est fermé, et la somme $F+G$ est topologique. On en déduit que $\operatorname{Im} T=(F+\operatorname{Ker} A) / \operatorname{Ker} A$ est fermée dans $E / \operatorname{Ker} A$, ce qui équivaut à l'existence d'une constante $C_{T}$ telle que (voir par exemple [8], Théorème 5.1, Page 70):

$$
\|y+F \cap \operatorname{Ker} A\| \leq C_{T}\|T y\|, \quad \text { pour tout } y \in F .
$$

De même, comme $\operatorname{Im} A$ est fermée, il existe une constante $C_{A}$ telle que:

$$
\|x+\operatorname{Ker} A\| \leq C_{A}\|A x\|, \quad \text { pour tout } x \in E .
$$

Donc, pour tout $y \in F$, on a:

$$
\begin{aligned}
\|y+\operatorname{Ker}(A \mid F)\|=\|y+F \cap \operatorname{Ker} A\| & \leq C_{T}\|T y\|=C_{T}\|y+\operatorname{Ker} A\| \\
& \leq C_{T} C_{A}\|A y\|=C_{T} C_{A}\|(A \mid F) y\| .
\end{aligned}
$$

Ainsi il existe une constante $C_{A \mid F}=C_{T} C_{A}$ telle que:

$$
\|y+\operatorname{Ker}(A \mid F)\| \leq C_{A \mid F}\|(A \mid F) y\|, \quad \text { pour tout } y \in F .
$$

Ceci traduit le fait que $\operatorname{Im}(A \mid F)$ est fermée dans $H$.

Le lemme suivant est facile à établir: 
Lemme 3.2. Soient $E$ un espace de Banach complexe et $A \in \mathcal{B}(E)$ tels que $E=E_{1} \oplus E_{2}$ avec $E_{1}, E_{2} \in$ LatA. Alors, on a:

$$
\operatorname{Im} A=\operatorname{Im} A_{1} \dot{+} \operatorname{Im} A_{2} \quad \text { et } \quad \overline{\operatorname{Im} A}=\overline{\operatorname{Im} A_{1}} \oplus \overline{\operatorname{Im} A_{2}},
$$

où $A_{1}=A\left|E_{1}, A_{2}=A\right| E_{2}$ et $\overline{\operatorname{Im} A}$ désigne la fermeture de $\operatorname{Im} A$ pour la topologie de la norme.

Définition 3.3. Soit $E$ un espace de Banach complexe. Un opérateur $A \in$ $\mathcal{B}(E)$ est dit à images des itérés fermées si $\operatorname{Im} A^{k}=A^{k} E$ est fermée, pour tout $k \in \mathcal{N}$.

Dans [4], M. El Oufir avait caractérisé les opérateurs nilpotents à images des itérés fermées dans un espace de Banach à l'aide des sous-espaces réduisants de dimension finie. Nous reprenons ce résultat en en donnant une démonstration plus rapide.

Théorème 3.4 (El Oufir). Soient $E$ un espace de Banach complexe et $A \in$ $\mathcal{B}(E)$ un opérateur nilpotent. Alors, les propriétés suivantes sont équivalentes:

(i) A est à images des itérés fermées.

(ii) Pour tout élément $x$ de E, il existe un sous-espace réduisant pour $A$ de dimension finie contenant $x$.

(iii) Pour tout sous-espace $F$ de $E$ de dimension finie, il existe un sousespace réduisant pour $A$ de dimension finie contenant $F$.

Démonstration. (i) $\Longrightarrow$ (ii): Si $x$ est nul, le résultat est trivial. Supposons $x$ non nul et posons $m=1+h\left(A^{k-1} x\right)$ où $k=e(x)$. Deux cas sont à distinguer:

1 er cas. Pour tout $j \leq k-1, h\left(A^{j} x\right)=j+h(x)$.

Ceci équivaut à $h(x)=m-k$. Soit $x_{1} \in E$ tel que $x=A^{m-k} x_{1}$. Alors, $e\left(x_{1}\right)=m$ et $A^{m-1} x_{1} \notin A^{m} E$. Comme $A^{m} E$ est fermée, alors, d'après le théorème de Hahn-Banach, il existe une forme linéaire $u$ continue sur $E$ telle que:

$$
u\left(A^{m} E\right)=0 \quad \text { et } \quad u\left(A^{i} x_{1}\right)=\delta_{m-1}^{i} \text { pour } \quad 0 \leq i \leq m-1,
$$

où $\delta$ désigne le symbole de Kronecker. On vérifie alors aisément que l'opérateur:

$$
B=\sum_{s=1}^{k} A^{s-1}\left(x_{1} \otimes u\right) A^{k-s}
$$

est continu sur $E$ d'image $\operatorname{Vect}_{A}\left(x_{1}\right)$ et qu'il commute avec $A$. Par suite, $\operatorname{Vect}_{A}\left(x_{1}\right)$ est réduisant pour $A$ et contient $\operatorname{Vect}_{A}(x)$.

2ème cas. Il existe $j \leq k-1$ tel que $h\left(A^{j} x\right)>j+h(x)$.

Soit $j_{1}=\min \left\{j \leq k-1 ; h\left(A^{j} x\right)>j+h(x)\right\}$. Pour $x^{\prime}=A^{k-j_{1}} x$, on est dans le premier cas $\left(e\left(x^{\prime}\right)=j_{1}\right.$ et $\left.h\left(x^{\prime}\right)=h\left(A^{j_{1}-1} x^{\prime}\right)+1-j_{1}\right)$, donc il 
existe $x_{1} \in E$ tel que $A^{m-j_{1}} x_{1}=A^{k-j_{1}} x$ et $\operatorname{Vect}_{A}\left(x_{1}\right)$ est réduisant pour $A$ contenant $\operatorname{Vect}_{A}\left(A^{k-j_{1}} x\right)$. Quitte à changer de générateur, on peut supposer que

$$
\operatorname{Vect}_{A}\left(x, x_{1}\right)=\operatorname{Vect}_{A}\left(x_{1}\right) \oplus \operatorname{Vect}_{A}(y)
$$

avec $y=x-A^{m-k} x_{1}$. Le vecteur $y$ est d'exposant $e(y)=k-j_{1}$. On passe alors à $y$. En poursuivant jusqu'au bout, on obtient un sous-espace $H=\oplus_{i=1}^{q} \operatorname{Vect}_{A}\left(x_{i}\right)$ réduisant pour $A$ et contenant $\operatorname{Vect}_{A}(x)$.

(ii) $\Longrightarrow$ (iii): Le résultat étant vrai pour $F=\{0\}$, supposons $\operatorname{dim} F>0$ et raisonnons par récurrence sur $\operatorname{dim} F$. Soit $x$ un élément non nul de $F$, alors d'après (ii), il existe un sous-espace $E_{1}$ réduisant pour $A$, de dimension finie et contenant $x$. Soit $E_{2} \in$ Lat $A$ tel que $E=E_{1} \oplus E_{2}$, alors $F \subseteq$ $\left(E_{1}+F\right)=E_{1} \oplus\left(\left(E_{1}+F\right) \cap E_{2}\right)$. Comme $E_{1} \cap F$ n'est pas réduit à $\{0\}$, on a $\operatorname{dim}\left(E_{1}+F\right)<\operatorname{dim} E_{1}+\operatorname{dim} F$, ce qui entraîne $\operatorname{dim}\left(\left(E_{1}+F\right) \cap E_{2}\right)<$ $\operatorname{dim} F . A \mid E_{2}$ étant à images des itérés fermées, alors, d'après l'hypothèse de récurrence, il existe $H_{2}$ et $G_{2}$ dans $\operatorname{Lat}\left(A \mid E_{2}\right)$ tels que:

$$
E_{2}=H_{2} \oplus G_{2}, \quad \operatorname{dim} H_{2}<+\infty \quad \text { et } \quad\left(E_{1}+F\right) \cap E_{2} \subseteq H_{2} .
$$

De plus, il est clair que $E=\left(E_{1} \oplus H_{2}\right) \oplus G_{2}$. Ainsi, le sous-espace $E_{1} \oplus H_{2}$ est réduisant pour $A$, de dimension finie et contient $F$.

(iii) $\Longrightarrow$ (i): Montrons d'abord que $\operatorname{Im} A=A(E)$ est fermée.

Soit $x \in \overline{\operatorname{Im} A}$, alors il existe un sous-espace $H$ réduisant pour $A$, de dimension finie et contenant $x$. Soit $G \in L a t A$ tel que $E=H \oplus G$. $H$ étant de dimension finie, $\operatorname{Im}(A \mid H)$ est aussi de dimension finie, donc est fermée. D'après le Lemme 3.2, on a $\overline{\operatorname{Im} A}=\operatorname{Im}(A \mid H) \oplus \overline{\operatorname{Im} A \mid G}$. Dans cette décomposition le vecteur $x$ s'écrit $x=y+z$ où $y \in \operatorname{Im}(A \mid H)$ et $z \in \overline{\operatorname{Im}(A \mid G)}$. L'unicité de la décomposition entraîne $x=y$ et $z=0$. Par conséquent, $\operatorname{Im} A$ est fermée.

Comme $L a t A \subseteq L a t A^{j}$, pour tout $j \in \mathcal{N}$, on montre de la même manière que les images $\operatorname{Im} A^{j}=A^{j} E$ sont fermées.

Théorème 3.5. Soient $E$ un espace de Banach complexe et $A \in \mathcal{B}(E)$ un opérateur nilpotent à images des itérés fermées. Alors, tout sous-espace invariant pour $A$ de codimension finie contient un sous-espace réduisant pour $A$ de codimension finie.

Démonstration. Soient $F \in L a t A$ de codimension finie dans $E$ et $G$ un supplémentaire algébrique quelconque de $F$ dans $E$. Notons que $G$ n'est pas nécessairement invariant pour $A$. Comme $G$ est de dimension finie, il résulte du caractère nilpotent de $A$ que $\operatorname{Vect}_{A}(G)$ est de dimension finie, et par suite $\operatorname{dim}\left(\operatorname{Vect}_{A}(G) \cap F\right)<+\infty$. D'après le Lemme 3.1, l'opérateur $A \mid F$ est à images des itérés fermées, donc, suite au théorème d'El Oufir (Théorème 3.4), il existe un sous-espace $H$ réduisant pour $A \mid F$, de dimension finie et contenant $\operatorname{Vect}_{A}(G) \cap F$. Soit $K \in \operatorname{Lat}(A \mid F)$ tel que $F=H \oplus K$. 
Posons $H^{\prime}=\operatorname{Vect}_{A}(G)+H$, alors:

$$
H^{\prime} \in \operatorname{Lat} A, \quad \operatorname{dim} H^{\prime}<+\infty \quad \text { et } \quad E=H^{\prime} \oplus K .
$$

Ainsi, $K$ est un sous-espace réduisant pour $A$, de codimension finie et contenu dans $F$.

Notation 3.6. Soit $A \in \mathcal{B}(E)$ un opérateur nilpotent. Pour $m \in \mathcal{N}$, on pose:

$$
f_{m}(E, A)=\operatorname{dim}\left(\operatorname{Ker} A \cap A^{m} E / \operatorname{Ker} A \cap A^{m+1} E\right) .
$$

En dimension finie, $f_{m}(E, A)$ est le nombre de sous-espaces cycliques de $E$ de dimension $m+1$ qui interviennent dans une décomposition de Jordan de $A$ dans $E$. Il est connu que si $E_{1}$ et $E_{2}$ sont deux $\mathcal{C}$-espaces vectoriels de dimension finie et si $A_{1}$ et $A_{2}$ sont deux opérateurs nilpotents respectivement sur $E_{1}$ et $E_{2}$, alors:

$$
\left(E_{1}, A_{1}\right) \cong\left(E_{2}, A_{2}\right) \Longleftrightarrow \forall m \in \mathcal{N}, f_{m}\left(E_{1}, A_{1}\right)=f_{m}\left(E_{2}, A_{2}\right) .
$$

Proposition 3.7. Soient $E$ un espace vectoriel de dimension finie sur $\mathcal{C}$, $A \in \mathcal{B}(E)$ un opérateur nilpotent et $E_{1}, E_{2}, F_{1}, F_{2}$ des sous-espaces invariants pour $A$ tels que:

$$
(E, A)=\left(E_{1}, A_{1}\right) \oplus\left(F_{1}, B_{1}\right)=\left(E_{2}, A_{2}\right) \oplus\left(F_{2}, B_{2}\right)
$$

où $A_{i}=A \mid E_{i}$ et $B_{i}=A \mid F_{i}(i=1,2)$.

Si $\left(E_{1}, A_{1}\right) \cong\left(E_{2}, A_{2}\right)$, alors $\left(F_{1}, B_{1}\right) \cong\left(F_{2}, B_{2}\right)$.

Démonstration. D'après (2), il suffit de vérifier que, pour tout $m \in \mathcal{N}$, on a:

$$
f_{m}(E, A)=f_{m}\left(E_{1}, A_{1}\right)+f_{m}\left(F_{1}, B_{1}\right) .
$$

Or $\operatorname{Ker} A \cap A^{m} E=\left(\operatorname{Ker} A_{1} \cap A_{1}^{m} E_{1}\right) \oplus\left(\operatorname{Ker} B_{1} \cap B_{1}^{m} F_{1}\right)$, donc en posant:

$$
U_{m}(E, A)=\left(\operatorname{Ker} A \cap A^{m} E\right) /\left(\operatorname{Ker} A \cap A^{m+1} E\right),
$$

on obtient l'isomorphisme de $\mathcal{C}$-espaces vectoriels suivant:

$$
U_{m}(E, A) \cong U_{m}\left(E_{1}, A_{1}\right) \oplus U_{m}\left(F_{1}, B_{1}\right) .
$$

L'égalité (3) s'en déduit facilement.

Le résultat de la Proposition 3.7 se généralise au cas des opérateurs nilpotents à images des itérés fermées dans un espace de Banach quelconque, et ce de la façon suivante:

Théorème 3.8. Soient $E$ un espace de Banach complexe, $A \in \mathcal{B}(E)$ nilpotent à images des itérés fermées et $E_{1}, E_{2}, F_{1}, F_{2}$ des sous-espaces invariants pour A tels que:

$$
(E, A)=\left(E_{1}, A_{1}\right) \oplus\left(F_{1}, B_{1}\right)=\left(E_{2}, A_{2}\right) \oplus\left(F_{2}, B_{2}\right)
$$

où $A_{i}=A \mid E_{i}$ et $B_{i}=A \mid F_{i}(i=1,2)$. 
Si $\left(E_{1}, A_{1}\right)$ et $\left(E_{2}, A_{2}\right)$ sont isomorphes et de dimension finie, alors $\left(F_{1}, B_{1}\right)$ et $\left(F_{2}, B_{2}\right)$ sont isomorphes.

\section{Démonstration.}

1ère étape. On va commencer par montrer que $E_{1}+E_{2}$ est contenu dans un sous-espace $G$ invariant pour $A$ de dimension finie, que $F_{1} \cap F_{2}$ contient un sous-espace $K$ invariant pour $A$ et que l'on a $E=G \oplus K$.

D'après le Lemme 3.1, $B_{1}=A \mid F_{1}$ est à images des itérés fermées. Comme $\operatorname{dim}\left(\operatorname{Vect}_{A}\left(E_{1}+E_{2}\right) \cap F_{1}\right)<+\infty$, il résulte du théorème d'El Oufir qu'il existe $H_{1}$ et $K_{1}$ dans $L a t B_{1}$ tels que:

$$
F_{1}=H_{1} \oplus K_{1}, \quad \operatorname{dim} H_{1}<+\infty \quad \text { et } \quad \operatorname{Vect}_{A}\left(E_{1}+E_{2}\right) \cap F_{1} \subseteq H_{1} .
$$

Posons $H_{1}^{\prime}=H_{1}+\operatorname{Vect}_{A}\left(E_{1}+E_{2}\right)$, alors:

$$
H_{1}^{\prime} \in \operatorname{Lat} A, \quad \operatorname{dim} H_{1}^{\prime}<+\infty \quad \text { et } \quad E=H_{1}^{\prime} \oplus K_{1} .
$$

D'après le Lemme 3.1, l'opérateur $A \mid K_{1}$ est à images des itérés fermées. Comme codim $\left(K_{1} \cap F_{2}\right)<+\infty$, alors il vient du Théorème 3.5. Qu'il existe un sous-espace $K$ réduisant pour $A \mid K_{1}$, de codimension finie (dans $K_{1}$ ) et contenu dans $K_{1} \cap F_{2}$. Soit $K_{2} \in \operatorname{Lat}\left(A \mid K_{1}\right)$ tel que $K_{1}=K_{2} \oplus K$, alors $E=\left(H_{1}^{\prime} \oplus K_{2}\right) \dot{+} K$. Comme $H_{1}^{\prime} \oplus K_{2}$ et $K$ sont deux sous-espaces (fermés !!) de $E$, leur somme directe est topologique. Aussi en posant $G=H_{1}^{\prime} \oplus K_{2}$, a-t-on:

$G, K \in L a t A, \operatorname{dim} G<+\infty, E_{1}+E_{2} \subseteq G, K \subseteq F_{1} \cap F_{2}$ et $E=G \oplus K$.

2ème étape. $\quad E_{1}$ est réduisant pour $A$, donc pur dans $(E, A)$ d'après le Lemme $2.2 \mathrm{Il}$ s'ensuit du Lemme 2.1 que $E_{1}$ est pur dans $(G, A \mid G)$. Comme $G$ est de dimension finie, le Lemme 2.3 entraîne que $E_{1}$ est réduisant pour $A \mid G$. On montre de même que $E_{2}$ est réduisant pour $A \mid G$. Soient donc $G_{1}$ et $G_{2}$ dans Lat $A$ tels que $G=E_{1} \oplus G_{1}=E_{2} \oplus G_{2}$, alors:

$$
(G, A \mid G)=\left(E_{1}, A_{1}\right) \oplus\left(G_{1}, C_{1}\right)=\left(E_{2}, A_{2}\right) \oplus\left(G_{2}, C_{2}\right)
$$

où $C_{i}=A \mid G_{i}(i=1,2)$.

Comme $\left(E_{1}, A_{1}\right) \cong\left(E_{2}, A_{2}\right)$, alors $\left(G_{1}, C_{1}\right) \cong\left(G_{2}, C_{2}\right)$ (d'après la Proposition 3.7) et il s'ensuit que:

$$
\left(G_{1}, C_{1}\right) \oplus(K, T) \cong\left(G_{2}, C_{2}\right) \oplus(K, T)
$$

où $T=A \mid K$.

D'autre part, des égalités (4) on déduit:

$$
(E, A)=\left(E_{1}, A_{1}\right) \oplus\left(G_{1}, C_{1}\right) \oplus(K, T)=\left(E_{2}, A_{2}\right) \oplus\left(G_{2}, C_{2}\right) \oplus(K, T) .
$$

Aussi a-t-on:

$$
\left.\begin{array}{l}
\left(G_{1}, C_{1}\right) \oplus(K, T) \cong(E, A) /\left(E_{1}, A_{1}\right) \cong\left(F_{1}, B_{1}\right) \\
\left(G_{2}, C_{2}\right) \oplus(K, T) \cong(E, A) /\left(E_{2}, A_{2}\right) \cong\left(F_{2}, B_{2}\right)
\end{array}\right\} .
$$

La conclusion $\left(F_{1}, B_{1}\right) \cong\left(F_{2}, B_{2}\right)$ découle immédiatement de (5) et (6). 
Remarque 3.9. Dans le théorème précédent, supposer uniquement $\left(E_{1}, A_{1}\right)$ isomorphe à $\left(E_{2}, A_{2}\right)$ sans que la dimension ne soit finie n'entraîne pas nécessairement l'existence d'un isomorphisme entre $\left(F_{1}, B_{1}\right)$ et $\left(F_{2}, B_{2}\right)$. En effet, soient $T \in \mathcal{B}(E)$ un opérateur nilpotent cyclique d'ordre $k \in \mathcal{N}^{*}$ et $(E, A)=\oplus_{i \in \mathcal{N}}\left(H_{i}, T_{i}\right)$ où $\left(H_{i}, T_{i}\right)=(H, T)$, pour tout $i \in \mathcal{N}$. Si on pose

$$
\left(E_{1}, A_{1}\right)=(E, A) \quad \text { et } \quad\left(E_{2}, A_{2}\right)=\oplus_{i=2}^{+\infty}\left(H_{i}, T_{i}\right)
$$

alors il est évident que $\left(E_{1}, A_{1}\right) \cong\left(E_{2}, A_{2}\right)$. Cependant,

$$
(E, A) /\left(E_{1}, A_{1}\right) \cong(\{0\}, 0) \quad \text { et } \quad(E, A) /\left(E_{2}, A_{2}\right) \cong(H, T) .
$$

\section{Extensions continues d'opérateurs nilpotents à images des itérés fermées par des opérateurs nilpotents "de dimension finie".}

Soient $E^{\prime}$ et $E^{\prime \prime}$ deux espaces de Banach complexes. Une extension continue de $A^{\prime} \in \mathcal{B}\left(E^{\prime}\right)$ par $A^{\prime \prime} \in \mathcal{B}\left(E^{\prime \prime}\right)$ est un opérateur $A \in \mathcal{B}(E)$ tel que le module $(E, A)$ soit une extension du module $\left(E^{\prime}, A^{\prime}\right)$ par le module $\left(E^{\prime \prime}, A^{\prime \prime}\right)$. Ceci s'exprime aussi par l'existence d'une suite exacte courte:

$$
0 \longrightarrow\left(E^{\prime}, A^{\prime}\right) \stackrel{\chi}{\longrightarrow}(E, A) \stackrel{\sigma}{\longrightarrow}\left(E^{\prime \prime}, A^{\prime \prime}\right) \longrightarrow 0 .
$$

Nous allons nous restreindre aux extensions $(E, A)$ telles que $E$ soit de la forme $E^{\prime} \oplus E^{\prime \prime}$, ce qui permet d'utiliser une représentation matricielle des opérateurs de $\mathcal{B}(E)$ (une telle restriction est assez naturelle car dans le cas des espaces hilbertiens on a toujours $E=E^{\prime} \oplus E^{\prime \prime}$-existence du complément orthogonal-). Un opérateur $A \in \mathcal{B}\left(E^{\prime} \oplus E^{\prime \prime}\right)$ est une extension de $A^{\prime} \in \mathcal{B}\left(E^{\prime}\right)$ par $A^{\prime \prime} \in \mathcal{B}\left(E^{\prime \prime}\right)$ si et seulement s'il existe un opérateur linéaire continu $B$ de $E^{\prime \prime}$ dans $E^{\prime}$ tel que la matrice de $A$ soit de la forme:

$$
A=\left[\begin{array}{cc}
A^{\prime} & B \\
0 & A^{\prime \prime}
\end{array}\right]
$$

Dans la suite, on suppose que $A^{\prime \prime} \in \mathcal{B}\left(E^{\prime \prime}\right)$ est "de dimension finie" (i.e., $E^{\prime \prime}$ est de dimension finie). Si $A \in \mathcal{B}(E)$ est une extension de $A^{\prime}$ par $A^{\prime \prime}$, alors $E^{\prime}$ est un sous-espace de $E$ de codimension finie, donc admet un supplémentaire topologique dans $E$. Ceci vient de l'isomorphisme $E^{\prime \prime} \cong E / E^{\prime}$ et du fait que $E^{\prime \prime}$ soit de dimension finie. En identifiant $E^{\prime \prime}$ à un supplémentaire de $E^{\prime}$ dans $E$, on peut écrire $E=E^{\prime} \oplus E^{\prime \prime}$. D'autre part, de l'exactitude de (7) on déduit qu'un changement de supplémentaire de $E^{\prime}$ dans $E=E^{\prime} \oplus E^{\prime \prime}$ laisse invariante la forme de Jordan de $A^{\prime \prime}$. Supposons en plus que $A^{\prime \prime} \in \mathcal{B}\left(E^{\prime \prime}\right)$ est nilpotent, alors quitte à changer, si besoin est, de supplémentaire de $E^{\prime}$ 
dans $E^{\prime} \oplus E^{\prime \prime}$, on peut supposer que l'extension $A$ vérifie:

$$
\begin{cases}E^{\prime \prime}=\oplus_{j=1}^{q} \operatorname{Vect}_{A^{\prime \prime}}\left(e_{j}\right) & \text { avec } e_{j} \text { d'exposant } n_{j}, \\ A^{s} e_{j}=A^{\prime \prime s} e_{j} & \text { pour } s<n_{j}, \\ A^{n_{j}} e_{j} \in E^{\prime} & \text { pour } 1 \leq j \leq q .\end{cases}
$$

On voit donc que l'étude des extensions de $A^{\prime} \in \mathcal{B}\left(E^{\prime}\right)$ par un opérateur nilpotent "de dimension finie" est liée à l'étude des sous-espaces réduisants pour $A^{\prime}$ minimaux contenant un sous-espace de dimension finie. Un cas qui serait intéressant à étudier est celui où $A^{\prime} \in \mathcal{B}\left(E^{\prime}\right)$ est nilpotent à images des itérés fermées.

Théorème 4.1. Soient $A^{\prime} \in \mathcal{B}\left(E^{\prime}\right)$ nilpotent à images des itérés fermées et $A^{\prime \prime} \in \mathcal{B}\left(E^{\prime \prime}\right)$ nilpotent "de dimension finie". Alors toute extension continue de $A^{\prime}$ par $A^{\prime \prime}$ est à images des itérés fermées.

Démonstration. Soit $A \in \mathcal{B}(E)$ une extension de $A^{\prime}$ par $A^{\prime \prime}$. D'après ce qui précède, on peut supposer $E=E^{\prime} \oplus E^{\prime \prime}$ et que $A$ vérifie (9). Comme $A^{\prime}$ est à images des itérés fermées, alors d'après le théorème d'El Oufir (Théorème 3.4), il existe un sous-espace $F^{\prime}$ réduisant pour $A^{\prime}$, de dimension finie et contenant $\operatorname{Vect}\left(A^{n_{1}} e_{1}, \cdots, A^{n_{q}} e_{q}\right)$. Soit $G^{\prime} \in \operatorname{Lat} A^{\prime}$ tel que $E^{\prime}=$ $G^{\prime} \oplus F^{\prime}$, alors $E^{\prime} \oplus E^{\prime \prime}=G^{\prime} \oplus\left(F^{\prime} \oplus E^{\prime \prime}\right)$. De plus, $G^{\prime}$ et $F^{\prime} \oplus E^{\prime \prime}$ étant dans Lat $A$, on a $A=A\left|G^{\prime} \oplus A\right|\left(F^{\prime} \oplus E^{\prime \prime}\right)$. Or, suite au Lemme 3.1, $A\left|G^{\prime}=A^{\prime}\right| G^{\prime}$ est à images des itérés fermées et, d'autre part, $F^{\prime} \oplus E^{\prime \prime}$ étant de dimension finie, $A \mid\left(F^{\prime} \oplus E^{\prime \prime}\right)$ est aussi à images des itérés fermées. Par conséquent, il résulte du Lemme 3.2 que $A$ est à images des itérés fermées.

Remarque 4.2. En général, une extension continue d'un opérateur nilpotent à images des itérés fermées n'est pas nécessairement à images des itérés fermées. En effet, soient $H^{\prime}$ et $H^{\prime \prime}$ deux espaces de Hilbert isomorphes à un même espace de Hilbert séparable, $\left(e_{n}\right)_{n \in \mathcal{N}^{*}}\left(\operatorname{resp} .\left(f_{n}\right)_{n \in \mathcal{N}^{*}}\right)$ une base hilbertienne de $H^{\prime}$ (resp. $\left.H^{\prime \prime}\right)$ et $A \in \mathcal{B}\left(H^{\prime} \oplus H^{\prime \prime}\right)$ défini pour tout $n \in \mathcal{N}^{*}$ par $A e_{n}=0$ et $A f_{n}=\frac{1}{n} e_{n}$. Alors $A$ est nilpotent d'ordre 2 à image $\operatorname{Im} A=A\left(H^{\prime \prime}\right)$ non fermée $\left(A\left(H^{\prime \prime}\right)\right.$ est dense dans $H^{\prime}$ mais distinct de $\left.H^{\prime}\right)$. Cependant, $A$ est une extension continue de l'endomorphisme nul (qui est à image fermée !) de $H^{\prime}$ par l'endomorphisme nul de $H^{\prime \prime}$ :

$$
A=\left[\begin{array}{cc}
0 & B \\
0 & 0
\end{array}\right]
$$

où $B$ est un opérateur continu de $H^{\prime \prime}$ dans $H^{\prime}$ défini par $B f_{n}=\frac{1}{n} e_{n}$.

Remarque 4.3. Soient $E$ un espace de Banach complexe, $A \in \mathcal{B}(E)$ un opérateur nilpotent à images des itérés fermées et $F$ un sous-espace de dimension finie dans $E$. Si $F^{\prime}$ est un sous-espace réduisant pour $A$ minimal 
contenant $F$, alors $F^{\prime}$ est de dimension finie et est unique à isomorphie près (voir K. Benabdallah et B. Charles [1]). Dans le cas où $F$ est cyclique ou lorsque $F$ est contenu dans le noyau de $A$, on démontre que le sous-module $\left(F^{\prime}, A \mid F^{\prime}\right)$ est unique à isomorphie près (voir respectivement [3] et [5]). Le cas où $F$ est quelconque reste cependant à étudier.

Application. Soient $A^{\prime} \in \mathcal{B}\left(E^{\prime}\right)$ nilpotent à images des itérés fermées, $A^{\prime \prime} \in \mathcal{B}\left(E^{\prime \prime}\right)$ un opérateur nilpotent "de dimension finie" et $A \in \mathcal{B}(E)$ une extension de $A^{\prime}$ par $A^{\prime \prime}$. Supposons que $A$ vérifie (10). Soit $F^{\prime}$ un sous-espace réduisant pour $A^{\prime}$ minimal contenant $A^{n_{1}} e_{1}, \cdots, A^{n_{q}} e_{q}$ et soit $G^{\prime} \in \operatorname{Lat} A^{\prime}$ tel que $E^{\prime}=G^{\prime} \oplus F^{\prime}$, alors:

$$
(E, A)=\left(G^{\prime}, A \mid G^{\prime}\right) \oplus\left(F^{\prime} \oplus E^{\prime \prime}, T\right)
$$

où $T=A \mid\left(F^{\prime} \oplus E^{\prime \prime}\right)$ est une extension de $A \mid F^{\prime}$ par $A^{\prime \prime}$.

Si $A^{\prime \prime}$ est cyclique (i.e., $\left.q=1\right)$, alors $\left(F^{\prime}, A^{\prime} \mid F^{\prime}\right)=\left(F^{\prime}, A \mid F^{\prime}\right)$ étant unique à isomorphie près, il en est de même pour $\left(F^{\prime} \oplus E^{\prime \prime}, T\right)$. Cela entraîne, d'après le Théorème 3.8 , que $\left(G^{\prime}, A \mid G^{\prime}\right)$ est aussi unique à isomorphie près. On en déduit que l'étude des extensions d'opérateurs nilpotents à images des itérés fermées par un opérateur nilpotent cyclique se ramène à l'étude en dimension finie des extensions d'opérateurs nilpotents par un opérateur nilpotent cyclique.

En procédant de la même façon, on se ramène aussi au cas de la dimension finie lorsque $q$ est quelconque et que $A^{n_{1}} e_{1}, \ldots, A^{n_{q}} e_{q}$ sont dans $\operatorname{Ker} A^{\prime}$.

Remarque 4.4. Nous nous sommes basés pour la définition des extensions d'opérateurs linéaires sur B. Charles [2].

Je tiens à exprimer ma profonde gratitude au Professeur B. Charles pour ses conseils judicieux et son aide efficace qui m'ont permis l'achèvement de ce travail.

\section{References}

[1] K. Benabdallah and B. Charles, Orbits of invariants subspaces of algebraic linear operators, Linear Algebra Appl., 225 (1995), 13-22, MR 97a:15005.

[2] B. Charles, Opérateurs linéaires sur un espace de Banach et modules sur un anneau principal, Symposia Mathematica, XIII (1979), 121-143, MR 82a:47018, Zbl 485.47009.

[3] B. Charles et A. Faouzi, Forme de Jordan des extensions d'opérateurs linéaires (Problème de Carlson) et sous-espaces réduisants minimaux contenant un sous-espace donné, Linear Algebra Appl., 288 (1999), 45-51, MR 99k:15021, Zbl 939.15005.

[4] M. El Oufir, Etude des sous-espaces réduisants de dimension finie d'un opérateur nilpotent sur un espace de Banach, Thèse de 3ème cycle, Université de Montpellier II, Octobre 1987 (texte multigraphié). 
[5] A. Faouzi, Sur la forme de Jordan des extensions d'opérateurs linéaires, II, preprint soumis pour publication.

[6] L. Fuchs, Infinite Abelian Groups, I, II, Academic Press, 1970; 1973, MR 41 \#333, MR 50 \#2362, Zbl 209.05503.

[7] I. Kaplansky, Infinite Abelian Groups, The University of Michigan (1954), MR 16,444g, Zbl 057.01901.

[8] M. Schechter, Principles of Functional Analysis, Academic Press, 1973, MR 57 \#7085, Zbl 211.14501.

Received September 16, 1999.

DÉPARTEMEnt De MAThÉmatiques et Informatique Université ChouaïB Doukkalli

FACulté Des Sciences, B.P.: 20

24000 EL JADIDA

MAROC

E-mail address: faouzi@ucd.ac.ma afaouzi@hotmail.com 